\title{
La médiation documentaire sur les réseaux socionumériques comme vecteur de l'e- réputation?
}

La Poste, Twitter et les agents-facilitateurs

\section{Camille Alloing}

\section{OpenEdition}

\section{Journals}

Édition électronique

URL : http://journals.openedition.org/communicationorganisation/4139

DOI : 10.4000/communicationorganisation.4139

ISSN : $1775-3546$

\section{Éditeur}

Presses universitaires de Bordeaux

\section{Édition imprimée}

Date de publication : 1 juin 2013

Pagination : 73-84

ISBN : 978-2-86781-883-7

ISSN : 1168-5549

\section{Référence électronique}

Camille Alloing, «La médiation documentaire sur les réseaux socionumériques comme vecteur de l'eréputation? ", Communication et organisation [En ligne], 43 | 2013, mis en ligne le 01 juin 2015, consulté le 30 avril 2019. URL : http://journals.openedition.org/communicationorganisation/4139; DOI :

10.4000/communicationorganisation.4139 


\title{
La médiation documentaire sur les réseaux socionumériques comme vecteur de l'e-réputation? La Poste, Twitter et les agents-facilitateurs
}

\author{
Comille Alloing'
}

\section{Contexte}

Le web dit « 2.0» (O’Reilly, 2005) ou « social» (Proulx et al., 2010) regroupe un ensemble de dispositifs sociotechniques (blogs, wikis, bookmarking, etc.) permettant aux « internautes » de communiquer, s'informer ou encore nouer des relations. Comme pour Internet en général, un certain imaginaire (Flichy, 2000) se développe progressivement autour de ce web social où l'utilisateur serait au centre du modèle, mais dont des analyses plus critiques soulèvent les visées économiques de plates-formes «enfermant » les internautes pour mieux canaliser leur attention, et monétiser leurs données personnelles et traces d'activités (Bouqillion et Matthews, 2010). Dans les discours des praticiens, les organisations sont alors fortement incitées à profiter de cette nouvelle ressource économique pour favoriser leurs actions d'information-communication (Le Deuff, 2006, A).

Ces dispositifs sont communément regroupés sous l'appellation, aux contours flous, de " médias sociaux ", dont l'un des derniers avatars sont les réseaux socionumériques (Stenger et Coutant, 2010), tels Facebook. com ou Twitter.com. Ces RSN sont des plates-formes où les internautes développent des profils publics ou semi-publics, qu'ils connectent ensuite à ceux d'autres utilisateurs (boyd et Ellison, 2007). Si ces dispositifs permettent la prise de contact avec d'autres individus, l'activité info-communicationnelle " ordinaire " relève de la prescription d'autres contacts ou de documents (Stenger et Coutant, 2009).

Ce profil sur un RSN développé par un internaute forme alors une « identité numérique » (Pierre, 2011). Pour Georges (2008, 09), une identité numérique repose sur l'agencement d'autres identités : une déclarative (ce que l'internaute dit de lui), une agissante (son agir sur les dispositifs) et une

1 Ingénieur R\&D La Poste, Doctorant en Sciences de l'Information et de la Communication au CEREGE (EA 1722) ; alloingcamille@gmail.com 
calculée (les computations effectuées par les systèmes informatiques régissant les plates-formes de RSN). Notons que l'identité numérique est multiple en fonction des supports, contextes et attributs qui les constituent (Coutant et Stenger, 2011) : un internaute a donc des identités numériques.

"Après avoir été pensée comme une cible, qui venait après une information déjà constituée, la personne est devenue une ressource, un agent de pertinence et un opérateur de liens entre les informations. " (Merzeau, 2009, B). Les attributs identitaires en ligne viennent personnaliser l'information prescrite, et participent à la computation des documents numériques (comme des pages web) pour leur mémorisation et mise en visibilité, ainsi que pour la hiérarchisation des résultats sur les moteurs de recherche ${ }^{2}$ afin de définir leur popularité voire leur «l'influence » ou celle de leurs auteurs (Le Deuff, 2006, B). De plus, ces internautes incarnés par leurs traces documentaires en ligne constitutives de leurs identités numériques participent à une « médiation identitaire » (Merzeau, 2012, A), c'est-à-dire à une légitimation de l'information diffusée en ligne mais aussi à une orientation dans le choix des ressources informationnelles pour les autres internautes. Les organisations quant à elles nous semblent développer une " présence numérique ", plus globale, et constituée autant de leurs profils que de l'ensemble des documents qu'elles diffusent ou qui lui sont rattachés.

Le développement de cette présence, et surtout sa gestion, nécessitent alors selon nous une forme de médiation documentaire définie ainsi par Liquète et al. (2010) : "La médiation, lorsqu'elle s'appuie sur des dispositifs matériels ou humains en capacité de lier information et communication, peut être qualifiée de médiation documentaire. ». Les dispositifs matériels sont ici les RSN, ceux humains les internautes et leur médiation identitaire et prescription ordinaire, l'organisation venant alors orienter au sein de sa présence ses publics par ces dispositifs dans une visée de communiquer avec ces dits publics. Lorganisation effectue ainsi une médiation des savoirs (Fabre et Gardiès, 2010) au quotidien : en encodant les connaissances sur elle-même et son environnement dans des documents numériques (vidéos, billets de blogs, tweets), puis en facilitant leur accès par les dispositifs sociotechniques où elle développe sa présence, elle produit un effet sur les connaissances que ses publics ont d'elle (et ce afin de mieux vendre un produit ou entrer en interaction par la suite).

Au-delà, l'ensemble des documents diffusés sur les RSN concernant l'organisation, lorsqu'ils véhiculent des évaluations sur l'être ou l'agir de celle-ci, et qu'ils sont vecteurs de la formulation d'une opinion, peuvent interroger leur impact sur la " réputation en ligne " des organisations. Nous questionnant alors : quelle(s) forme(s) de médiation(s) documentaire(s) une organisation doit-elle effectuer sur les réseaux socionumériques qu'elle souhaite investir afin de construire ou gérer sa réputation? Dans cet article, nous proposons

2 Google appellerait cette nouvelle forme de pondération "l'AuthorRank » (en référence au Pagerank), cf. http://descary.com/preparez-vous-a-larrivee-de-lauthorrank-wordpress 
tout d'abord de définir plus avant la notion de " réputation en ligne » ou "e-réputation », puis nous exposons une méthodologie mise en place afin de favoriser cette médiation documentaire sur les RSN, en l'occurrence ici pour La Poste et sur Twitter.

\section{De la réputation à l'e-réputation des organisations}

En Sciences Humaines les approches de la réputation des organisations sont variées. Pour les économistes, la réputation est un mécanisme d'approbation (Smith, 1759), autant qu'un signal visant à réduire l'incertitude des actions et conduites futures d'un acteur en situation de choix (Milgrom et Roberts, 1986). Pour les gestionnaires, la réputation est « l'image globale » (association des différentes « images mentales » propres à l'individu) que se font l'ensemble des "parties prenantes » d'une organisation à partir de ses actions passées et des informations produites à son sujet (Fombrun et Van Riel, 1997). La réputation d'une organisation repose donc sur certains éléments rationnels (informations que l'on peut capter), et sur un traitement aussi bien cognitif qu'affectif (image). En sociologie, la réputation est un processus social amenant à la production de sceaux d'approbations (Origgi, 2007) agissant sur le statut, les interactions et la représentation sociale d'une entité (individu ou organisation).

En SIC, Origgi (2007) aborde la réputation comme " la valeur informationnelle de nos actions ». Pour d'Almeida (2007), la réputation s'inscrit dans une théorie du jugement : elle cristallise en quelque sorte l'ensemble des opinions et des jugements portés sur les actions d'une entité. Allant plus loin, nous pouvons dire que la réputation est une méta-opinion : une opinion formulée à partir de jugements de faits, ou de croyances, portant sur d'autres opinions exprimées (et notamment présentent dans les informations diffusées à l'encontre d'une entité). D'un point de vue de la gestion de la réputation d'une organisation, la communication s'avère centrale (Boistel, 2008) : elle repose sur l'identité de l'organisation (ce qu'elle souhaite montrer) et suppose pour l'organisation des actes de communication visant à produire des "signaux de conformité " qui seront diffusés par les bons "filtres " (journalistes par exemple) venant les crédibiliser, et ce afin de développer une congruence communicationnelle entre les valeurs de l'organisation et celles de son environnement ou de celui de ses publics (Philippe et Durand, 2009).

$\mathrm{Si}$ nous transposons la réputation au web, quid de cette " réputation en ligne »? De nombreux chercheurs, principalement anglo-saxons, abordent cette online reputation de manière instrumentale, soit comme l'ensemble des systèmes informatiques visant à générer de la confiance sur les sites marchands, à hiérarchiser les informations, et spécifiquement sur des sites comme Ebay.com (McDonald et Slawson, 2000). En 2001, Chun et Davies parlent « d'e-réputation » pour désigner cette réputation en ligne, et pointent l'importance de la diffusion de signaux de conformité emprunts 
des valeurs de l'organisation sur son site web. L'e-réputation devient par la suite un terme fortement employé en France par les praticiens pour désigner l'ensemble des facteurs et actions visant à développer et assurer la réputation d'une organisation par le biais du web social (Fillias et Villeneuve, 2011). Approches opératoires transposant la réputation au web (" l'image que se font les internautes ») et s'appuyant fortement sur « l'imaginaire » du web 2.0 (type «les internautes sont au pouvoir»). En SIC, l'e-réputation n'est que peu abordée par le prisme des organisations mais est généralement questionnée au travers des identités numériques d'un individu (Galinon-Mélénec, 2011).

Pour mieux circonscrire cette notion d'e-réputation, nous avons proposé (Alloing et Pierre, 2012) le cadre théorique en SIC suivant : « L'e-réputation procède d'une redocumentarisation itérative automatisée ou endogène des interprétations et connaissances produites en ligne par les individus ou les organisations, forgeant un attribut identitaire servant de marqueur prescriptif destiné à cadrer dans un contexte asynchrone et atopique les formes d'engagement ultérieures, et facilitée par les fonctionnalités techniques des sites web où elle opère une informatisation du microsocial au profit et avec les moyens de la logique marchande ". L'e-réputation donc, comme redocumentarisation (Zacklad, 2006) des traces et des informations produites par les internautes, mais aussi des documents inscrivant les savoirs portant sur l'être et l'agir d'une entité. Cette redocumentarisation a visée prescriptive apparait comme une forme de médiation du document effectuée non-plus par le professionnel du document, mais par l'usager lui-même (Liquète et al., 2010) afin de produire des indicateurs de pertinence, et agissant sur les trois dimensions du document numérique (Pédauque, 2003) : le vu (réarticulation de la forme d'un document), le lu (réarticulation du sens), et le su (personnalisation par les attributs identitaires).

\section{Médiation documentaire et e-réputation}

À partir de ce contexte et de cette approche de l'e-réputation, nous pouvons ici formuler l'hypothèse suivante : pour construire ou gérer son e-réputation sur et par les RSN, l'organisation doit développer une forme de médiation documentaire. En somme, faciliter l'accès aux documents numériques porteurs de savoirs à son sujet et des valeurs qu'elle souhaite véhiculer, et ce en adaptant leur diffusion aux pratiques des internautes : par la production et la personnalisation de ces documents afin de permettre des formes de redocumentarisation propres à ses publics, en orientant les internautes vers les documents en pertinences avec leurs interrogations, ou encore en s'appropriant les dispositifs sociotechniques comme les blogs ou les RSN afin d'en faire des espaces documentaires en adéquation avec les besoins de ses publics et ses propres objectifs. L'organisation peut pour cela identifier les informations à inscrire dans les documents qu'elle diffuse, c'est-à-dire celles porteuses de signaux de conformité et participant à la formulation d'une opinion. Elle 
doit alors structurer son environnement informationnel, soit identifier les informations et documents présents sur les RSN et traitant des thématiques qu'elle veut aborder afin d'y circonscrire les bons signaux, ainsi que la manière dont ces documents sont prescrits afin d'en favoriser la diffusion. Voire, identifier les bons filtres dans le but de se reposer sur leur propre médiation documentaire et identitaire. Nous souhaitons donc maintenant présenter la mise en place et les premiers résultats d'une méthodologie visant à favoriser cette structuration de l'environnement informationnel.

\section{Structurer l'environnement informationnel pour construire les discours et favoriser la médiation : La Poste, Twitter et les agents-facilitateurs ${ }^{3}$}

La Loi « Grenelle 2 » $^{4}$ incite les organisations à améliorer leur politique de Responsabilité Sociale d'Entreprise (RSE). Les organisations, ici La Poste métier Courrier, peuvent alors profiter de la mise en place de ces directives pour développer leur « image verte » (Pascual Espuny, 2011). Les RSN sont un canal de cette communication environnementale, et interrogent La Poste sur les discours à produire et à diffuser (par le biais de documents ad hoc) afin de développer cette image verte tout en préservant la (e)réputation qu'elle souhaite avoir.

Dans le cadre de nos recherches, nous avons spécifiquement identifié sur Twitter (où la présence numérique de La Poste fait l'objet d'un développement $\mathrm{accru}^{5}$ ) des internautes dont la prescription documentaire les insère dans une forme "d'économie de la recommandation »(Pierre, 2011). Nous avons nommé ces internautes " agents-facilitateurs » (Alloing et Deschamps, 2011 ; Alloing, 2011, 2012), car : comme agents ils participent activement au fonctionnement d'un système informatique (le RSN), ils facilitent l'accès aux documents pour d'autres utilisateurs (ils collectent, contextualisent et diffusent de l'information en fonction de leur audience et de ses pratiques), ils permettent de sélectionner l'information sans l'emprise des dispositifs web dont le fonctionnement en «boîte noire » (Rieder, 2010 ) questionne la pertinence des résultats et analyses, ils offrent à l'organisation la possibilité d'analyser l'information par le prisme des internautes (ce qui leur semble pertinent ou non), ou encore ils participent à la mise en visibilité de certains documents.

À la suite de l'observation d'un lancement de produit sur le web par La Poste (Alloing, 2011), nous avons établi une typologie spécifique à Twitter de ces agents, à partir de 61 profils et en fonction de 44 critères : les attributs identitaires (comme la présence d'une biographie ou non, du volume d'abonnés,

\footnotetext{
3 Ces résultats s'inscrivent dans le cadre d'une recherche doctorale financée et menée au sein de la Direction du Système d'Information de La Poste métier Courrier.

4 Voir : http://www.legrenelle-environnement.fr/Presentation-de-la-loi-Grenelle-2.html

5 Voir les comptes@lisalaposte (assistance aux clients), @Groupelaposte (communication dite corporate) ou encore @musedelaposte (évènements liés au Musée de La Poste).
} 
d'une identité civile), les pratiques informationnelles (ajouts de métadonnées pour personnaliser l'information comme les " hashtags " [\#], tendances à reprendre un message d'un autre compte ou à en produire un original), et la capacité de médiatisation (nombreuses reprises des messages, volume de citations du profil). Cette typologie, constituée du croisement inductif de ces critères puis de l'observation du traitement de l'information effectué par les profils ainsi catégorisés, ne se veut en rien prédictive quant aux comportements futurs, mais permet d'identifier des pratiques types pouvant par la suite être intégrées aux actions de collecte d'informations de l'organisation.

Dans le cadre des actions de communication sur la RSE de La Poste nous avons proposé un système de collecte d'information basé sur les agents-facilitateurs, et visant à répondre à deux questions principales : quelles informations concernant la thématique sont diffusées aux publics de La Poste sur le web (afin de construire les discours de l'organisation) ? Et quelle forme de médiation documentaire est effectuée pour prescrire ces informations, et dont La Poste pourrait ensuite s'inspirer pour faciliter l'accès à ses publics aux documents qu'elle produit ? Pour cela, nous avons tout d'abord interviewé la collaboratrice responsable de la communication sur ce sujet, et définit les thématiques à aborder (4 thématiques principales, 27 sous-thématiques), le volume d'informations à lui fournir, les valeurs que La Poste souhaite véhiculer sur ces sujets (comme la confiance, l'innovation, la proximité), et le type de traitement de l'information (personnalisation forte ou non). L'étape suivante, en juillet 2012, a consisté à sélectionner des agents-facilitateurs. Pour identifier les internautes publics de La Poste et prescrivant des informations liées au RSE nous nous somme basés sur un corpus de 15 articles traitant de la « lettre verte » de La Poste, considérant que « en laissant un contenu informationnel sur le Net [ou en le relayant], l'internaute présuppose un lecteur et cette présupposition induit le type de contenu qu'il envoie sur le Net ", (Monseigne et al., 2011), en l'occurrence ici des publics intéressés par certaines actions de La Poste sur ces sujets. Nous avons ainsi identifié 78 relais dont les comptes Twitter étaient actifs les trois derniers mois. Puis nous les avons catégorisés en fonction de notre typologie, pour obtenir au final 34 comptes pertinents, répartis en quatre catégories : experts ${ }^{7}$, veilleurs ${ }^{8}$, collecteurs $^{9}$ et passionnés $^{10}$.

\footnotetext{
6 À l'aide de l'outil topsy.com

7 Critères principaux : identité civile affichée, plus grand volume d'abonnés que d'abonnements, production plutôt que relai de messages, fort volume de liens hypertextes dans les messages diffusés (par rapport à l'ensemble des comptes), forte utilisation des hashtags, rythme constant de diffusion, diffusion d'informations ou de sources « expertes » (spécialisées sur un sujet)...

8 Identité civile affichée, biographie présentant les thématiques abordées, plus grand volume d'abonnements que d'abonnés, relai « automatique » (à l'aide du bouton Twitter dédié) plutôt que production de messages, rythme de diffusion en adéquation avec l'actualité...

9 Pseudonyme, faible volume d'abonnements et volume d'abonnés variable, fort volume de messages publiés, quasi-totalité des messages contenant un lien hypertexte, pas ou peu d'échanges avec les autres usagers de Twitter, automatisation d'une partie de la diffusion des messages, forte utilisation des hashtags, rythme intensif de diffusion...

10 Pseudonyme, biographie « anecdotique » (ou humoristique), plus d'abonnements que d'abonnés (ou part
} 
Le dispositif suivant a été développé afin de collecter l'information :

- Création d'un compte Twitter ${ }^{11}$ afin de s'abonner aux comptes, et ainsi pouvoir observer directement les pratiques ;

- Mise en place d'un flux RSS puis d'un filtre de celui-ci avec l'outil pipes. yahoo.com en fonction des mots-clés, et pour supprimer tout message ne contenant pas de liens hypertextes ;

- L'outil Yahoo Pipes nous a ensuite permis de construire un nouveau flux RSS que nous avons intégré à la plate-forme Infomous.com. Infomous regroupe les principales données issues d'un flux RSS par « clusters sémantiques » (Figure 1) interactifs (chaque terme rassemble des liens vers les documents).

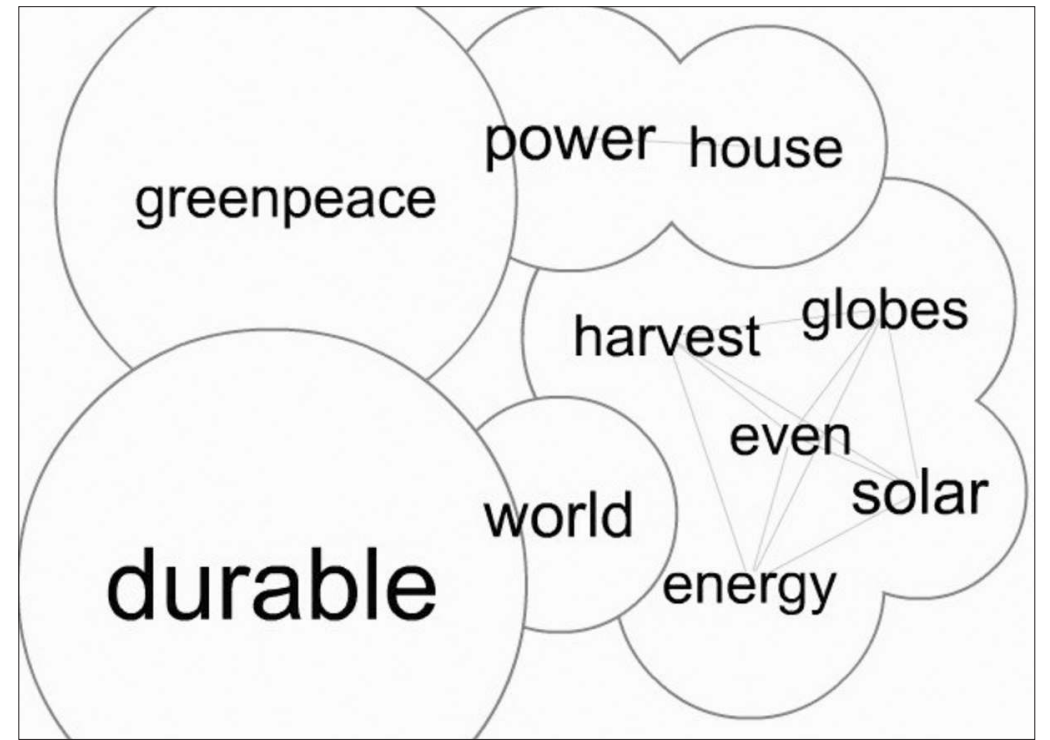

Figure 1 : Exemple d'une cartographie interactive réalisée avec Infomous.com le 28/08/12

\section{Résultats et discussion}

Durant deux mois (juillet à septembre 2012) nous avons collecté au travers de notre dispositif près de 1200 messages renvoyant vers 750 sources ou documents différents (billets de blogs essentiellement -dont 1/4 en anglais). Ces messages (tweets) peuvent être appréhendés en fonction de leurs modèles de publication (Rebillard et Charton, 2004) :

- Messages contenant un lien vers un document numérique (article de presse, de blog, vidéo) et accompagnés du titre de celui-ci -modèle éditorial ;

égal), converse plus que ne diffuse des informations ou des liens vers des documents, utilisation de hashtags " clair-obscur » (dont la signification fait référence à des échanges précédents)...

11 http://twitter.com/GreenVeille 
- Messages contenant un lien vers un document et un ajout de métadonnées (hashtag, commentaire) - niveau méta-éditorial ;

- Messages reprenant un autre message (retweet) avec ajout ou non de métadonnées - publication distribuée (au niveau du RSN).

Les principaux sujets récurrents étant la gestion des déchets, la communication environnementale des organisations ou encore les véhicules électriques. Le dispositif nous a offert l'occasion d'identifier cinq sources de référence par sujets ${ }^{12}$, permettant ainsi à La Poste de définir par la suite les principaux filtres de ces futurs documents et messages. De même, certaines informations pouvant être jugées comme « critiques » par La Poste et ses publics (changement de réglementation par exemple) ont été détectées.

Dans une optique de construction de discours en congruence avec l'environnement informationnel des publics, le dispositif a mis en exergue le fait que les informations concernant le RSE sont principalement " expertes » (très détaillées et en provenance de professionnels du sujet), que ces informations sont très fortement argumentées et peu orientées vers un public profane, que la vulgarisation de ces informations autant que leur médiatisation incombe généralement aux ONG telles Greenpeace voire à certains médias généralistes, ou encore que des sujet prêtent fortement à débat ou à la formulation quasi-systématique d'opinions dépréciatives. De plus, nos observations ont donné lieu à recommandation quant aux actions de médiation documentaire que La Poste pourrait effectuer depuis ses comptes Twitter : les documents produits doivent être argumentés, ou encore certaines métadonnées (hashtags) permettent de mieux contextualiser (à l'échelle de Twitter) les messages envoyés (comme \#green ou \#carbone).

Enfin, ces observations donnent une typologie des publics en fonction des discours présents dans les documents relayés : le public non spécialiste des questions de RSE diffuse et discute les actions des organisations concernant les « grandes causes » (gestion du Co2 par exemple); les publics passionnés ou engagés se focalisent sur le volontarisme des organisations (prise de position affirmée) ; les publics experts ou professionnels relaient des informations démontrant l'aspect innovant et performatif des actions mises en place.

Globalement, cette médiation documentaire effectuée par les agents-facilitateurs par et sur les RSN offre donc à La Poste la possibilité de définir des discours en fonction des publics, d'identifier les types d'informations en adéquation avec les valeurs qu'elle a pu définir, et de repérer de possibles affordances situationnelles amenant à une action rapide et limitée dans le temps. De manière générale, La Poste peut ainsi amorcer les bases d'une construction proactive de son e-réputation par la mise en adéquation de ses discours et de ses futures actions de médiations avec les pratiques, les

12 Car les plus prescrites sur l'ensemble des sources identifiées, mais aussi les plus discutées par les internautes (commentaires suite aux publications). 
attentes et l'environnement informationnel de ses publics. Plus que capter l'attention de ses publics, l'organisation peut alors l'orienter au sein des RSN où se construit sa présence documentaire numérique...

\section{Conclusion}

En guise de conclusion, nous pouvons tout d'abord souligner que l'e-réputation d'une organisation sur les RSN s'appuie sur la médiation documentaire : pour lier l'information produite à son encontre à la communication qu'elle souhaite développer, et comme moyen et processus de collecte des signaux informationnels pertinents pour ses publics par l'identification notamment d'agents-facilitateurs. Ensuite, plus qu'une surveillance distante ou une captation décontextualisée et sous l'emprise des dispositifs sociotechniques des données personnelles ou traces d'usages des internautes, l'organisation doit tendre vers le développement de liens de confiance avec les internautes pourvoyeurs de documents porteurs d'opinions : plutôt que surveiller, elle doit " prendre soin » de ces ressources (Le Deuff, 2009). En clair, viser une forme de " pharmacologie informationnelle » afin d'éviter toute surcharge cognitive et accroitre ses capacités décisionnelles et communicationnelles. Enfin, construire son e-réputation semble moins relever de l'accroissement d'une possible "influence ", souvent recherchée par les praticiens, que du développement à plus long terme de son autorité informationnelle (Broudoux, 2007) : l'organisation doit définir les processus lui permettant de donner forme, transmettre et légitimer les informations la concernant. Mais si ces informations permettent de formuler une opinion, et que les computations des dispositifs transforment ces opinions en indicateurs, ne serait-il pas alors plus pertinent, pour une organisation, de supposer qu'elle doive accroître son « autorité réputationnelle »?

\section{BIBLIOGRAPHIE}

ALLOING C., DESCHAMPS. C., «Veille stratégique et Internet participatif : les usages des agents-facilitateurs remettent-ils en question le concept de signal faible ? ", Colloque Spécialisé en Sciences de l'information: Management de l'information, défis et tendances, Université de Moncton, Canada, juin 2011.

ALLOING C., "Curation sociale et agents-facilitateurs : quel(s) impact(s)sur les stratégies d'information et de communication des organisations sur le web ? ", Médias011: Y a-t-il une richesse des réseaux, Aix-en-Provence, décembre 2011, en ligne $:$ http://hal. archives-ouvertes.fr/hal-00651333.

ALLOING C., PIERRE J., "Construire un cadre d'analyse avec les SIC pour comprendre les pratiques et les enjeux de la réputation en ligne (des individus et des organisations) ", XVIII Congrès de la Société Française des Sciences de l'Information et de la 
Communication, Rennes, 30 mai et 1er juin 2012, en ligne : http://hal.archives-ouvertes. fr/hal-00704007.

ALLOING C., «De surveiller à « prendre soin »: comment repenser la veille sur les réseaux sociaux numériques en termes de management de réseaux d'acteurs ? ", Revue Internationale d'Intelligence Economique, 1/2012 (Vol.4), p. 55-70.

d'ALMEIDA N., La société du jugement: Essai sur les nouveaux pouvoirs de l'opinion, Armand Colin, 2007.

BOISTEL P., « La réputation d'entreprise : un impact majeur sur les ressources de l'entreprise ", Management E̋ Avenir, 2008/3 n 17, p. 9-25.

BOUQUILLION P., MATTHEWS J.T., Le Web collaboratif. Mutations des industries de la culture et de la communication, Presses universitaires de Grenoble, 2010.

BOYD D., ELLISON N., " Social Network Sites: Definition, History, and Scholarship ", Journal of Computer-Mediated Communication, vol. 13, 2007, en ligne : http://jcmc.indiana.edu/vol13/issue1/boyd.ellison.html.

BROUDOUX E., «Construction de l'autorité informationnelle sur le web », 2007, en ligne : http://archivesic.ccsd.cnrs.fr/sic_00120710_v1.

CHARTRON G., REBILLARD F, Modèles de publication sur le web - Rapport final AS 103, CNRS/Département STIC/RTP 33 «Documents et contenus : création, indexation, navigation », 2004, en ligne : http ://archivesic.ccsd.cnrs.fr/sic_00001159.

CHUN R., DAVIES G., «E-reputation: The role of mission and vision statements in positioning strategy », The Journal of Brand Management, vol. 8, 2001, p. 315-333.

COUTANT A., STENGER T., " Production et gestion d'attributs identitaires ", Les Cabiers du numérique, 2011/1 Vol. 7, p. 61-74, 2011.

FABRE I., GARDIES C., "La médiation documentaire ", in Médiations, LIQUETE V. Dir., CNRS Editions, 2010, p. 121-140.

FILIAS E. VILLENEUVE A., E-réputation. Stratégies d'influence sur Internet, Ellipses, 2011.

FLICHY P., «Technologies fin de siècle : l'Internet et la radio ", Réseaux, vol. 18 n'100, 2000, p. 249-271.

FOMBRUN C., VAN RIEL C., "The Reputational Landscape ", Corporate Reputation Review, vol.1, 1997, p. 5-13.

GALINON-MÉLÉNEC B. dir., L'Homme trace. Perspectives antbropologiques des traces contemporaines, Paris, CNRS éditions, 2011.

GEORGES F., «Les composantes de l'identité dans le web 2.0, une étude sémiotique et statistique. Hypostase de l'immédiateté ", Actes du 76ème congrès de l'ACFAS: Web participatif: mutation de la communication, Institut national de la recherche scientifique, Québec, 6-7 mai 2008.

GEORGES F., «Représentation de soi et identité numérique. Une approche sémiotique et quantitative de l'emprise culturelle du web 2.0 ", Réseaux, 2009/2, n 154, p. $165-193,2009$.

LE DEUFF O. [A], « Le succès du web 2.0 : histoire, techniques et controverse ", 2006, en ligne : http://archivesic.ccsd.cnrs.fr/sic_00133571. 
LE DEUFF O. [B], «Autorité et pertinence vs popularité et influence : réseaux sociaux sur Internet et mutations institutionnelles », en ligne $:$ http://archivesic.ccsd.cnrs. fr/sic_00122603, 2006.

LE DEUFF O., «Le réveil de la veille : prendre soin plutôt que surveiller », Intercdi, 2009, n²20, p. 66-68.

LIQUETE V., FABRE I., GARDIES C., « Faut-il reconsidérer la médiation documentaire ? ", Les Enjeux de l'information et de la communication, 2010, p. 43-57.

MCDONALD C.G., SLAWSON V.C., " Reputation in an Internet Auction Market », Social Science Research Network, 21 mars 2000.

MERZEAU L., "Présence numérique : les médiations de l'identité ", article en ligne : http://archivesic.ccsd.cnrs.fr/docs/00/48/32/93/PDF/ENJEUX-MERZEAU. pdf, 2009.

MERZEAU L. [A], " La médiation identitaire ", Revue française des sciences de l'information et de la communication, 2012, en ligne : http://rfsic.revues.org/193.

MERZEAU L. [B], "Faire mémoire de nos traces numériques », E-dossiers de l'audiovisuel, 2012, en ligne : http://halshs.archives-ouvertes.fr/halshs-00727308.

MILGROM P., ROBERTS J., " Price and Advertising Signals of Product Quality », Journal of Political Economy, vol. 94, 1986, p. 796-821.

MONSEIGNE A., GALINON-MELENEC B., « La sémiotique des "signes-traces" appliquée au recrutement : le cas de la recherche du "bon candidat" via les traces numériques ", Communication et organisation, 2011.

O'REILLY T., "What Is Web 2.0. Design Patterns and Business Models for the Next Generation of Software », 2005, en ligne : http://oreilly.com/web2/archive/ what-is-web-20.html.

ORIGGI G., «Un certain regard. Pour une épistémologie de la réputation », Worshop «Réputation», Fondazione Olivetti, Roma ,14 avril 2007.

PASCUAL ESPUNY C., "Comment les organisations se saisissent-elles de 1' "image verte" ? ", Communication E organisation, vol. 34, 2008, en ligne : http:// communicationorganisation.revues.org/572.

PEDAUQUE R.T., « Document : forme, signe et médium, les re-formulations du numérique », 08 juillet 2003, en ligne : http://archivesic.ccsd.cnrs.fr/sic_00000511.html

PHILIPPE D., DURAND R., « Communication environnementale et réputation de l'organisation ", Revue française de gestion, 2009/4 n 194, p. 45-63.

PIERRE J., "Génétique de l'identité numérique. Sources et enjeux des processus associés à l'identité numérique ", Les Cabiers du numérique, 2011/1 Vol. 7, p. 15-29, 2011.

PROULX S., MILLERAND F., RUEFFJ., Web Social:Mutation de la Communication, PUQ, 2010.

RIEDER B., « Pratiques informationnelles et analyse des traces numériques : de la représentation à l'intervention ", Études de communication, vol. 35, 2010.

SMITH A., The Theory of Moral Sentiments, 1759, en ligne : http://www.ibiblio.org/ ml/libri/s/SmithA_MoralSentiments_p.pdf. 
STENGER T., COUTANT A., « La prescription ordinaire sur les réseaux socionumériques ", Médias 09, entre communautés et mobilité, Aix-en-Provence, 2009, en ligne : http://hal.archives-ouvertes.fr/hal-00458319.

STENGER T., COUTANT A., «Vers un management des « amis » sur les réseaux socionumériques?» 15ème colloque del 'Association Information E Management (AIM 2010), La Rochelle : France, 2010, en ligne : http://hal.archives-ouvertes.fr/hal-00525841.

WENG L., MENCZER F., "Context Visualization for Social Bookmark Management », arXiv:1211.6799, en ligne : http://arxiv.org/abs/1211.6799.

ZACKLAD M., «Une approche communicationnelle et documentaire des TIC dans la coordination et la régulation des flux transactionnels », Document de travail, 2006, en ligne : http://archivesic.ccsd.cnrs.fr.

Résumé : Dans cet article nous abordons la gestion et la construction de l'e-réputation (ou réputation en ligne) d'une organisation par le prisme de la médiation documentaire. Nous proposons une méthodologie de structuration de l'environnement informationnel d'une organisation sur les réseaux socionumériques s'appuyant sur les actions de prescriptions informationnels d'internautes qualifiés d'agents-facilitateurs, et permettant à une organisation d'identifier des signaux de conformité et de construire des discours en congruence avec les opinions et attentes de ses publics. Nous appliquons cette approche au cas de La Poste métier Courrier, sur Twitter, et pour la thématique de la Responsabilité Sociale d'Entreprise (RSE).

Mots-clés : e-reputation, agents-facilitateurs, médiation documentaire, réseaux socionumeriques, Twitter.

Abstract : In this article we discuss the management and construction of e-reputation (or online reputation) of an organization through the prism of documentary mediation. We propose a methodology of informational structuration for firms on social networks based on informational recommendation by internet users, which we called facilitator-agents, and which permit to firm to identify signals of compliance and build discourses in adequation with opinions and expectations of their audiences. We apply this approach to the case of La Poste, Twitter, and the topic of Corporate Social Responsibility (CSR).

Keywords : E-Reputation, Facilitators-Agents, Documentary Mediation, Social Networks, Twitter. 